\title{
UPLC-MS/MS Simultaneous Determination of Methamphetamine, Amphetamine, Morphine, Monoacetylmorphine, Ketamine, Norketamine, MDMA, and MDA in Hair
}

\author{
Siyuan Chen ${ }^{1,2}$, Jianshe Ma ${ }^{1}$, Xianqin Wang ${ }^{1,2^{* *}}$ and Peiwu Geng ${ }^{3 *}$ \\ 'School of Pharmaceutical Sciences, Wenzhou Medical University, Wenzhou 325035, China \\ Institute of Forensic Science, Wenzhou Medical University, Wenzhou 325035, China \\ ${ }^{3}$ Laboratory of Clinical Pharmacy, The People's Hospital of Lishui, Lishui 323000, China
}

Received: 06 March 2019; accepted: 10 April 2019

\begin{abstract}
Hair is a stable specimen and has a longer detection window (from weeks to months) than blood and urine. Through the analysis of hair, the long-term information of the drug use of the identified person could be explored. Our work is to establish an ultra-performance liquid chromatography-tandem mass spectroscopy (UPLC-MS/MS) method for simultaneous determination of methamphetamine, amphetamine, morphine, monoacetylmorphine, ketamine, norketamine, 3,4-methylenedioxymethamphetamine (MDMA), and 3,4-methylenedioxyamphetamine (MDA) in hair. Methoxyphenamine was used as an internal standard. The chromatographic separation was performed on a UPLC ethylene bridged hybrid (BEH) C18 $(2.1 \mathrm{~mm} \times 50 \mathrm{~mm}, 1.7 \mu \mathrm{m})$ column using a mobile phase of acetonitrile-water with $10 \mathrm{mmol} / \mathrm{L}$ ammonium acetate solution which containing $0.05 \%$ ammonium hydroxide. The multiple reaction monitoring in positive electrospray ionization was used for quantitative determination. The intra-day and inter-day precisions (relative standard deviation [RSD]) were below $15 \%$. The accuracy ranged between $85.5 \%$ and $110.4 \%$, the average recovery rate was above $72.9 \%$, and the matrix effect ranged between $92.7 \%$ and $109.2 \%$. Standard curves were in the range of $0.05-5.0 \mathrm{ng} / \mathrm{mg}$, and the correlation coefficients were greater than 0.995 . The established UPLC-MS/MS method was applied to analyze the hair samples successfully.
\end{abstract}

Keywords: UPLC-MS/MS, methamphetamine, amphetamine, morphine, monoacetylmorphine, ketamine, norketamine, MDMA, MDA, hair

\section{Introduction}

Hair is used as a biological material and has an irreplaceable advantage over blood and urine [1-3]. Hair is a stable specimen and has a longer detection window (from weeks to months) than blood and urine [4-6]. Through the analysis of hair, could explore the long-term information of the drug use of the identified person. In particular, segmental hair analysis can provide useful information about drug abuse status and abuse history $[6,7]$. Therefore, hair analysis has immeasurable value for forensic applications.

Because of the complex matrix of hair samples, the analysis is susceptible to interference from endogenous substances, so measuring drug abuse in hair is a challenge for analytical laboratories [8-10]. Gas chromatography-mass spectrometry (GC-MS) has been reported to detect drugs in hair samples. However, GC-MS technology has the following disadvantages: sensitivity is usually insufficient, sample pretreatment takes a long time, and derivatization steps such as morphine are required for compounds with poor gas chromatographic behavior. Compared with GC-MS, liquid chromatography with MS (LC-MS) has great advantages in biological sample analysis $[11,12]$. Its use in forensic science is first directed to specific low-concentration polar analytes. In LC-MS analysis, the sensitivity is high, the amount of sample required is small, the sample pretreatment is simple, and no derivatization is re-

*Authors for correspondence: These authors contributed equally to this work lankywang@163.com (X.W.), gengpeiwu@163.com (P.G.). quired. Further, LC with tandem mass spectroscopy (LC-MS/ MS) overcomes matrix background interference and improves signal-to-noise ratio, so it is more sensitive to complex biological samples and more suitable for analysis of low-level components.

The purpose of this study was to develop and validate an analytical method for the determination of methamphetamine, amphetamine, morphine, monoacetylmorphine, ketamine, norketamine, 3,4-methylenedioxymethamphetamine (MDMA), and 3,4-methylenedioxyamphetamine (MDA) in human hair based on simple hair extraction, followed by ultra-performance liquid chromatography-tandem mass spectroscopy (UPLC-MS/MS) analysis. The main metabolite of methamphetamine was amphetamine; the main metabolites of heroin were morphine and monoacetylmorphine; the main metabolite of ketamine was norketamine; the main metabolite of MDMA was MDA. The selected toxicants represent the most common drugs such as methamphetamine, heroin, ketamine, and MDMA.

\section{Materials and Methods}

Chemical Reagents. Methamphetamine, amphetamine, morphine, monoacetylmorphine, ketamine, norketamine, MDMA, MDA, and methoxyphenamine (internal standard) (all purity $>98 \%$ ) were purchased from Sigma-Aldrich (Lewis, USA). Chromatographic grade acetonitrile and methanol were purchased from Merck (Darmstadt, Germany). Ultra-pure water (resistance $>18 \mathrm{M} \Omega$ ) was prepared by Milli-Q purification system (Bedford, USA).

This is an open-access article distributed under the terms of the Creative Commons Attribution-NonCommercial 4.0 International License (https://creativecommons.org/licenses/by-nc/4.0/), which permits unrestricted use, distribution, and reproduction in any medium for non-commercial purposes, provided the original author and source are credited, a link to the CC License is provided, and changes - if any - are indicated. 
Table 1. MRM parameters of the drugs

\begin{tabular}{lcccc}
\hline Compound & $\begin{array}{c}\text { Parent } \\
\text { ion }\end{array}$ & $\begin{array}{c}\text { Daughter } \\
\text { ion }\end{array}$ & $\begin{array}{c}\text { Cone } \\
\text { voltage }\end{array}$ & $\begin{array}{c}\text { Collision } \\
\text { voltage }\end{array}$ \\
\hline Methamphetamine & 150.1 & 91.1 & 15 & 35 \\
Amphetamine & 150.1 & 119.1 & 15 & 10 \\
Morphine & 136.1 & 91.1 & 20 & 20 \\
& 136.1 & 119.1 & 20 & 10 \\
6-Acetylmorphine & 286.2 & 153.1 & 55 & 40 \\
Ketamine & 286.2 & 165.2 & 55 & 40 \\
& 328.1 & 165.3 & 46 & 28 \\
Norketamine & 328.1 & 211.3 & 46 & 24 \\
& 238.0 & 124.9 & 28 & 26 \\
MDMA & 238.0 & 179.0 & 28 & 16 \\
& 224.1 & 125.1 & 40 & 32 \\
MDA & 224.1 & 207.1 & 40 & 12 \\
& 194.3 & 105.1 & 10 & 25 \\
& 194.3 & 163.2 & 10 & 15 \\
& 180.2 & 105.1 & 10 & 25 \\
& 180.2 & 163.2 & 10 & 15 \\
\hline
\end{tabular}

Instruments and Conditions. ACQUITY UPLC and XevoXevo TQ-S Micro triple quadrupole mass spectrometer (Waters Corporation, USA) were use for determination of drugs in hair. An UPLC BEH C18 $(2.1 \mathrm{~mm} \times 50 \mathrm{~mm}, 1.7 \mu \mathrm{m})$ column with a column temperature of $40{ }^{\circ} \mathrm{C}$ was used, and the mobile phase was acetonitrile $-10 \mathrm{mmol} / \mathrm{L}$ ammonium acetate solution (containing $0.05 \%$ ammonium hydroxide) at a flow rate of $0.4 \mathrm{~mL} / \mathrm{min}$ with an injection volume of $1 \mu \mathrm{L}$. Gradient elution is with $10 \%$ initial acetonitrile for $0.2 \mathrm{~min}$, increased into $80 \%$ in $1.3 \mathrm{~min}$, keep at $80 \%$ for $0.5 \mathrm{~min}$, then drop to $10 \%$ in $0.5 \mathrm{~min}$, and hold for $1.5 \mathrm{~min}$, with a total run time of $4.0 \mathrm{~min}$.

Nitrogen was used as desolvation gas $(800 \mathrm{~L} / \mathrm{h})$ and cone gas $(50 \mathrm{~L} / \mathrm{h})$. A capillary voltage of $2.5 \mathrm{kV}$, a source temperature of $150{ }^{\circ} \mathrm{C}$, and a desolvation temperature of $400{ }^{\circ} \mathrm{C}$ were used. Multiple reaction monitoring (MRM) was used for quantitative analysis (Table 1).

Standard Solution Preparation. Methamphetamine, amphetamine, morphine, monoacetylmorphine, ketamine, norketamine, MDMA, and MDA were formulated as a $100 \mu \mathrm{g} / \mathrm{mL}$ methanol stock solution. The stock solution was diluted with methanol to the working solution, and all solutions are stored in a $4{ }^{\circ} \mathrm{C}$ freezer.

Preparation for Standard Curve. Healthy human hairs were given appropriate amounts of methamphetamine, amphetamine, morphine, monoacetylmorphine, ketamine, norketamine, MDMA, and MDA working solutions, constructed in the range of $0.02-5.0 \mathrm{ng} / \mathrm{mg}$ hair standard solutions (0.02 $0.050 .2,1.0,2.0$, and $5.0 \mathrm{ng} / \mathrm{mg})$. Quality control samples at concentrations of $0.05,0.2,0.8$ and $4.0 \mathrm{ng} /$ mg were prepared using the same method.

Sample Processing. A 10-mg hair was weighed, and washed with ultrapure water and acetone, then dried, cut, and ground, $0.3 \mathrm{~mL}$ methanol added, subjected to ultrasonic water bath for $1 \mathrm{~h}$, with $13,000 \mathrm{rpm}$ at $4{ }^{\circ} \mathrm{C}$, and centrifuge for $10 \mathrm{~min} ; 100 \mu \mathrm{L}$ of the supernatant was transferred into a liner tube of a vial, and $1 \mu \mathrm{L}$ was used for UPLC-MS/MS analysis.

Method Validation. The bioanalysis method validation was established according to the guidance of the US Food and Drug Administration. Validation projects include selectivity, matrix effects, linearity, precision, accuracy, recovery, and stability [13-22].

Applications. In 2018, the laboratory received 1193 hair samples and established test methods for common drug screening analysis.

\section{Results and Discussion}

Method Optimization. The hair pretreatment in this study used a simple pretreatment with water and acetone. Designed to meet the needs of fast hair treatment, it also cleans the drugs attached to the hair surface, as well as dust, inorganic salts, oils, and other impurities.

Various methods for extracting related drugs from hair have been reported, including enzymatic hydrolysis, acid hydrolysis, and alkaline hydrolysis. For example, the extraction of amphetamines and ketamine drugs is usually carried out by sodium hydroxide hydrolysis. Morphine is extracted by acidhydrolysis, ultrasound-assisted, and liquid-liquid extraction methods, which has a long-term problem and cannot meet the needs of rapid experimental testing. In the direct methanol ultrasonic method, the measured drug is directly extracted with methanol by a simple dissolution extraction method. This reduces both the pre-processing time and the detection requirements.

As far as possible, the internal interfering substances are separated from the retention time by high-performance liquid chromatography (HPLC), and the mobile phase and chromatographic column determine the chromatographic behavior $[23,24]$. We tried different chromatographic columns such as $\mathrm{BEH} \mathrm{C} 18(2.1 \mathrm{~mm} \times 50 \mathrm{~mm} 1.7 \mu \mathrm{m}), \quad \mathrm{BEH} \quad \mathrm{C} 18$

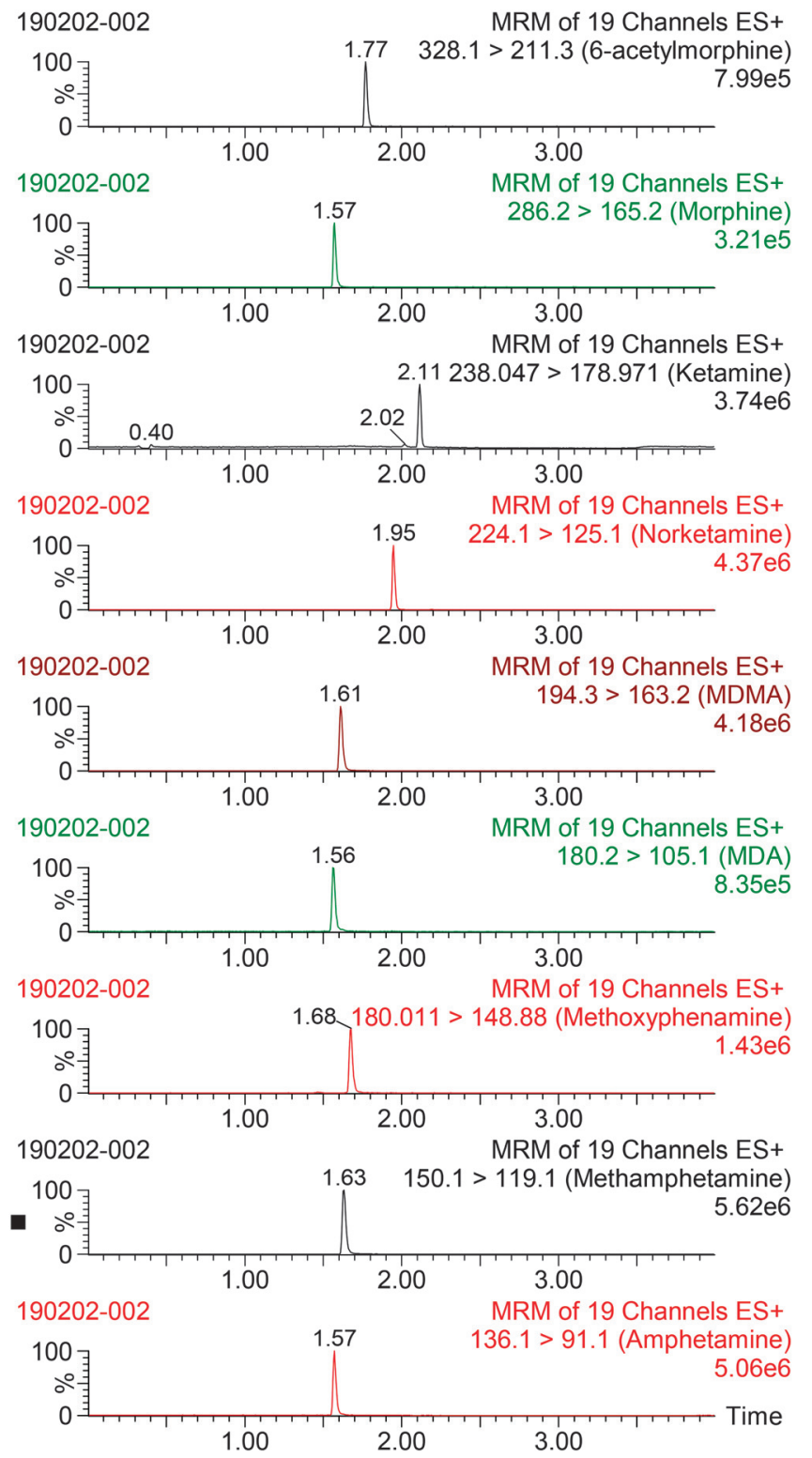

Figure 1. UPLC-MS/MS chromatograms of methamphetamine, amphetamine, morphine, monoacetylmorphine, ketamine, norketamine, MDMA, MDA, and methoxyphenamine (IS) in hair 
Table 2. Linear equations, correlation coefficient, LLOQ, and LOD of drugs in hair (LLOQ = lower limit of quantification; LOD = limit of detection)

\begin{tabular}{|c|c|c|c|c|c|}
\hline Compound & Linear equations & Concentration range (ng/mg) & Coefficient & LLOQ (ng/mg) & LOD (ng/mg) \\
\hline Methamphetamine & $y=9.8349 x+0.3089$ & $0.05-5.0$ & 0.9987 & 0.05 & 0.02 \\
\hline Amphetamine & $y=14.201 x+0.5447$ & $0.05-5.0$ & 0.9979 & 0.05 & 0.02 \\
\hline Morphine & $y=0.9067 x+0.0391$ & $0.05-5.0$ & 0.9978 & 0.05 & 0.02 \\
\hline Ketamine & $y=4.8998 x+0.1402$ & $0.05-5.0$ & 0.9993 & 0.05 & 0.02 \\
\hline Norketamine & $y=3.9917 x+0.0751$ & $0.05-5.0$ & 0.9997 & 0.05 & 0.02 \\
\hline MDMA & $y=12.296 x+0.4018$ & $0.05-5.0$ & 0.9976 & 0.05 & 0.02 \\
\hline MDA & $y=13.101 x-0.169$ & $0.05-5.0$ & 0.9997 & 0.05 & 0.02 \\
\hline
\end{tabular}

$(2.1 \mathrm{~mm} \times 100 \mathrm{~mm} 1.7 \mu \mathrm{m})$, and HSS T3 $(2.1 \mathrm{~mm} \times$ $100 \mathrm{~mm} 1.8 \mu \mathrm{m})$, and the results showed that $\mathrm{BEH} \mathrm{C18}$ $(2.1 \mathrm{~mm} \times 50 \mathrm{~mm} 1.7 \mu \mathrm{m})$ had the best peak time and peak effect. We tried acetonitrile- $0.1 \%$ formic acid, acetonitrile$10 \mathrm{mmol} / \mathrm{L}$ ammonium acetate solution (containing $0.1 \%$ formic acid), methanol- $0.1 \%$ formic acid, methanol-10 $\mathrm{mmol} / \mathrm{L}$ ammonium acetate solution (containing $0.1 \%$ formic acid), and acetonitrile- $-10 \mathrm{mmol} / \mathrm{L}$ ammonium acetate solution (containing $0.05 \%$ ammonium hydroxide) with gradient elution. The results showed that acetonitrile- $10 \mathrm{mmol} / \mathrm{L}$ ammonium acetate solution (containing $0.05 \%$ ammonium hydroxide) results in the most satisfactory chromatographic peak shape and retention time. Thus, BEH C18 $(2.1 \mathrm{~mm} * 50 \mathrm{~mm}, 1.7 \mu \mathrm{m})$ column and acetonitrile-10 $\mathrm{mmol} / \mathrm{L}$ ammonium acetate solution (containing $0.05 \%$ ammonium hydroxide) as the mobile phase were used in this work.

Method Validation. No impurities or endogenous substances that would interfere with the test could be identified, indicating that this method had good selectivity (Figure 1).

The equation for the standard curve of drugs was shown in Table 2. The intra-day precision RSD was below $15 \%$, and the inter-day precision RSD was below $15 \%$. The accuracy ranged between $85.5 \%$ and $110.4 \%$, the average recovery rate was above $72.9 \%$, and the matrix effect ranged between $92.7 \%$ and $109.2 \%$ (Table 3). The above results met the requirements of determination of drugs in biological tissues (acceptance criteria, intra-day, and interday accuracy: $\pm 15 \%$ of the nominal concentrations; except $\pm 20 \%$ at lower limit of quantification (LLOQ); intra-day and inter-day precision: $\pm 15 \% \mathrm{RSD}$, except $\pm 20 \% \mathrm{RSD}$ at LLOQ) [25].

UPLC-MS/MS was faster than traditional HPLC analysis and with enhanced signals [26-33]. Just $4 \mathrm{~min}$ to complete the analysis of plasma samples can save a lot of time. In addition, LLOQ $(0.05 \mathrm{ng} / \mathrm{mg})$ was relatively low, which can be used to determine low concentrations in hair.

Applications. In the laboratory, 1193 cases were detected. Positive concentration was set at $0.2 \mathrm{ng} / \mathrm{mg}$. In fact, 381 cases were methamphetamine, followed by 6-acetylmorphine, ketamine, and MDMA (Table 4). When methamphetamine was positive, the methamphetamine and amphetamine were simultaneously detected at the same time. When heroin was positive, 6-acetylmorphine and morphine were simultaneously detected. When ketamine was positive, ketamine and norketamine were simultaneously detected. When MDMA was positive, MDMA and MDA were simultaneous detected.

Table 3. Precision, accuracy, matrix effect, and recovery of drugs

\begin{tabular}{|c|c|c|c|c|c|c|c|}
\hline \multirow[t]{2}{*}{ Compound } & \multirow[t]{2}{*}{ Concentration (ng/mg) } & \multicolumn{2}{|c|}{ Precision (\% RSD) } & \multicolumn{2}{|c|}{ Accuracy (\%) } & \multirow[t]{2}{*}{ Matrix effect (\%) } & \multirow[t]{2}{*}{ Recovery (\%) } \\
\hline & & Intra-day & $\overline{\text { Inter-day }}$ & Intra-day & $\overline{\text { Inter-day }}$ & & \\
\hline \multirow{4}{*}{ Methamphetamine } & 0.05 & 13.1 & 12.8 & 106.0 & 85.5 & 97.4 & 89.4 \\
\hline & 0.2 & 4.3 & 9.0 & 108.2 & 102.3 & 100.3 & 74.0 \\
\hline & 0.8 & 5.5 & 10.5 & 100.8 & 94.9 & 101.6 & 76.2 \\
\hline & 4.0 & 8.1 & 7.1 & 103.1 & 96.3 & 93.7 & 86.1 \\
\hline \multirow{4}{*}{ Amphetamine } & 0.05 & 13.5 & 14.3 & 86.4 & 102.9 & 94.9 & 81.0 \\
\hline & 0.2 & 7.6 & 10.3 & 105.5 & 97.8 & 104.7 & 78.1 \\
\hline & 0.8 & 5.0 & 2.6 & 102.2 & 96.2 & 100.9 & 82.3 \\
\hline & 4.0 & 6.8 & 3.3 & 101.0 & 96.3 & 102.7 & 80.9 \\
\hline \multirow{4}{*}{ Morphine } & 0.05 & 12.8 & 13.6 & 91.2 & 93.3 & 96.6 & 73.1 \\
\hline & 0.2 & 10.6 & 11.9 & 100.1 & 106.1 & 92.7 & 86.6 \\
\hline & 0.8 & 8.6 & 5.3 & 96.0 & 91.5 & 89.8 & 82.9 \\
\hline & 4.0 & 4.6 & 5.6 & 100.7 & 94.2 & 94.9 & 72.9 \\
\hline \multirow{4}{*}{ 6-Acetylmorphine } & 0.05 & 14.3 & 14.7 & 110.4 & 88.5 & 96.2 & 83.0 \\
\hline & 0.2 & 7.3 & 12.6 & 103.2 & 104.7 & 93.3 & 84.3 \\
\hline & 0.8 & 11.2 & 9.8 & 95.1 & 108.0 & 97.6 & 83.0 \\
\hline & 4.0 & 10.3 & 10.9 & 99.9 & 101.2 & 92.4 & 89.5 \\
\hline \multirow{3}{*}{ Ketamine } & 0.2 & 5.9 & 9.3 & 106.9 & 97.0 & 107.9 & 79.8 \\
\hline & 0.8 & 6.8 & 5.8 & 92.8 & 86.5 & 101.8 & 75.2 \\
\hline & 4.0 & 5.5 & 4.7 & 106.8 & 109.3 & 105.5 & 85.8 \\
\hline \multirow{4}{*}{ Norketamine } & 0.05 & 12.4 & 14.2 & 102.8 & 102.2 & 95.2 & 92.2 \\
\hline & 0.2 & 9.7 & 12.0 & 94.1 & 97.4 & 90.0 & 95.4 \\
\hline & 0.8 & 4.3 & 10.9 & 100.7 & 98.3 & 98.3 & 95.0 \\
\hline & 4.0 & 2.5 & 4.6 & 102.3 & 97.3 & 104.1 & 91.8 \\
\hline \multirow{4}{*}{ MDMA } & 0.05 & 13.5 & 13.3 & 98.1 & 104.1 & 94.4 & 81.5 \\
\hline & 0.2 & 11.5 & 9.5 & 93.1 & 100.0 & 104.4 & 93.9 \\
\hline & 0.8 & 4.6 & 2.5 & 103.2 & 99.7 & 99.9 & 82.8 \\
\hline & 4.0 & 5.0 & 9.4 & 103.6 & 87.1 & 109.2 & 83.0 \\
\hline \multirow{4}{*}{ MDA } & 0.05 & 14.9 & 13.4 & 104.5 & 88.5 & 103.4 & 80.2 \\
\hline & 0.2 & 9.6 & 15.0 & 96.5 & 109.8 & 102.5 & 87.9 \\
\hline & 0.8 & 4.6 & 3.0 & 98.3 & 106.2 & 97.2 & 79.7 \\
\hline & 4.0 & 6.1 & 4.4 & 96.7 & 93.3 & 100.1 & 81.9 \\
\hline
\end{tabular}


Table 4. Application to determinate the hair sample in Wenzhou

\begin{tabular}{|c|c|c|c|c|c|c|c|c|c|c|c|}
\hline \multirow{2}{*}{$\begin{array}{l}\text { Area of } \\
\text { inspection }\end{array}$} & \multirow{2}{*}{$\begin{array}{l}\text { Number of } \\
\text { inspection }\end{array}$} & \multirow{2}{*}{$\begin{array}{l}\text { Positive } \\
\text { number }\end{array}$} & \multirow{2}{*}{$\begin{array}{l}\text { Positive } \\
\text { rate } \%\end{array}$} & \multicolumn{2}{|c|}{ Methamphetamine } & \multicolumn{2}{|l|}{ Heroin } & \multicolumn{2}{|c|}{ Ketamine } & \multicolumn{2}{|c|}{ MDMA } \\
\hline & & & & Methamphetamine & Amphetamine & 6-Acetylmorphine & Morphine & Ketamine & Norketamine & MDMA & $\overline{\mathrm{MDA}}$ \\
\hline Yueqing & 287 & 75 & 26.13 & 63 & 63 & 12 & 12 & & & & \\
\hline Ouhai & 247 & 82 & 33.20 & 65 & 65 & 18 & 18 & 6 & 6 & & \\
\hline Lucheng & 113 & 48 & 42.48 & 42 & 42 & 4 & 4 & 3 & 3 & & \\
\hline Cangnang & 113 & 36 & 31.86 & 32 & 32 & 1 & 1 & 3 & 3 & & \\
\hline Kaifaqu & 103 & 17 & 16.50 & 16 & 16 & 1 & 1 & & & & \\
\hline Yongjia & 98 & 25 & 25.51 & 19 & 19 & 7 & 7 & & & & \\
\hline Ruian & 79 & 38 & 48.10 & 27 & 27 & 1 & 1 & 10 & 10 & 11 & 11 \\
\hline Taishun & 56 & 26 & 46.43 & 26 & 26 & & & & & & \\
\hline Longwan & 40 & 14 & 35.00 & 6 & 6 & 1 & 1 & 8 & 8 & 1 & 1 \\
\hline Dongtou & 27 & 6 & 22.22 & 6 & 6 & & & & & & \\
\hline Wencheng & 24 & 9 & 37.50 & 5 & 5 & & & 2 & 2 & 3 & 3 \\
\hline Pingyang & 6 & 5 & 83.33 & 4 & 4 & & & 1 & 1 & & \\
\hline Total & 1193 & 381 & 31.94 & 311 & & 45 & & & 33 & 15 & \\
\hline
\end{tabular}

Positive concentration of methamphetamine, 6-acetylmorphine, morphine, ketamine, and MDMA was set at $0.2 \mathrm{ng} / \mathrm{mg}$, and the limit of detection of amphetamine, norketamine, and MDA was $0.02 \mathrm{ng} / \mathrm{mg}$.

\section{Conclusion}

In this study, a simple, rapid, and selective simultaneous determination of methamphetamine, amphetamine, morphine, monoacetylmorphine, ketamine, norketamine, MDMA, and MDA in hair by UPLC-MS/MS was developed and successfully applied to analysis of hair samples.

Acknowledgements. This work was supported by grants from the start-up funding from Wenzhou Medical University (QTJ17018).

\section{Reference}

1. Allibe, N.; Kintz, P.; Faure, A.; Paysant, F.; Michard-Lenoir, A. P.; Stanke-Labesque, F.; Scolan, V.; Eysseric-Guerin, H. Curr. Pharm. Des. 2017, 23, 5502-5510.

2. Wang, X. Drummer, O. H. Forensic Sci Int 2015, 257, 458-472.

3. Xiang, P.; Shen, M.; Drummer, O. H. J. Forensic Leg. Med. 2015, 36, $126-135$.

4. Vogliardi, S.; Tucci, M.; Stocchero, G.; Ferrara, S. D.; Favretto, D. Anal. Chim. Acta 2015, 857, 1-27.

5. Curtis, J.; Greenberg, M. Clin. Toxicol. 2008, 46, 22-34.

6. Srogi, K. Anal. Lett. 2006, 39, 231-258.

7. Srogi, K. Microchim. Acta 2006, 154, 191-212.

8. Kuwayama, K.; Nariai, M.; Miyaguchi, H.; Iwata, Y. T.; Kanamori, T.; Tsujikawa, K.; Yamamuro, T.; Segawa, H.; Abe, H.; Iwase, H.; Inoue, H. Int. J. Leg. Med. 2019, 133, 117-122.

9. Wu, Y.; Yang, J.; Duan, C. L.; Chu, L. X.; Chen, S. H.; Qiao, S.; Li, X. M.; Deng, H. H. J. Chromatogr. B: Anal. Technol. Biomed. Life Sci. 2018, 1083, 209-221.

10. Wang, X.; Johansen, S. S.; Nielsen, M. K. K.; Linnet, K. Forensic Sci. Int. 2018, 285, E1-E12.

11. Leung, K. W.; Wong, Z. C. F.; Ho, J. Y. M.; Yip, A. W. S.; Cheung, J. K. H.; Ho, K. K. L.; Duan, R.; Tsim, K. W. K. Drug Test. Anal. 2018, 10, 977-983.

12. Kronstrand, R.; Forsman, M.; Roman, M. Forensic Sci. Int. 2018, 283, 9-15.

13. Guo, J. Y.; Xu, Q. Q.; Tong, S. H.; Wang, S. H.; Zhang, Q. W.; Sun, F.; Wang, X. Q. Lat. Am. J. Pharm. 2014, 33, 1567-1570.
14. Cai, J. Z.; Huang, Z. Z.; Chen, X. L.; Xu, R. A.; He, H. Z.; Lin, C. L.; Lin, G. Y.; Wang, X. Q. Lat. Am. J. Pharm. 2012, 31, 388-393.

15. Ye, W.; Chen, R.; Sun, W.; Huang, C.; Lin, X.; Dong, Y.; Wen, C.; Wang, X. J. Chromatogr. B: Anal. Technol. Biomed. Life Sci. 2017, 1060, $144-149$.

16. Wen, C. C.; Wang, S. H.; Huang, X. L.; Liu, Z. Z.; Lin, Y. Y.; Yang, S. P.; Ma, J. S.; Zhou, Y. F.; Wang, X. Q. Biomed. Chromatogr. 2015, 29, $1805-1810$

17. Wen, C.; Zhang, Q.; He, Y.; Deng, M.; Wang, X.; Ma, J. Acta Chromatogr. 2015, 27, 81-91.

18. Wang, S. H.; Wu, H. Y.; Huang, X. L.; Geng, P. W.; Wen, C. C.; Ma, J. S.; Zhou, Y. F.; Wang, X. Q. J. Chromatogr. B: Anal. Technol. Biomed. Life Sci. 2015, 990, 118-124.

19. Tian, W. Q.; Cai, J. Z.; Xu, Y. Y.; Luo, X. H.; Zhang, J.; Zhang, Z. X.; Zhang, Q. W.; Wang, X. Q.; Hu, L. F.; Lin, G. Y. Int. J. Clin. Exp. Med. 2015, 8, 15164-15172.

20. Ma, J. S.; Wang, S. H.; Zhang, M. L.; Zhang, Q. W.; Zhou, Y. F.; Lin, C. L.; Lin, G. Y.; Wang, X. Q. Biomed. Chromatogr. 2015, 29, 1203-1212.

21. Ma, J. S.; Wang, S. H.; Huang, X. L.; Geng, P. W.; Wen, C. C.; Zhou, Y. F.; Yu, L. S.; Wang, X. Q. J. Pharm. Biomed. Anal. 2015, 111, 131-137.

22. Zhang, Q. W.; Wen, C. C.; Xiang, Z.; Ma, J. S.; Wang, X. Q. J. Pharm. Biomed. Anal. 2014, 90, 134-138.

23. Chen, L. G.; Su, K.; Jiang, Y. Y.; Chen, B. B.; Wen, C. C.; Zeng, M.; Wang, X. C. Lat. Am. J. Pharm. 2016, 35, 1450-1453.

24. Wang, X.; Zheng, M.; Liu, J.; Huang, Z.; Bai, Y.; Ren, Z.; Wang, Z.; Tian, Y.; Qiao, Z.; Liu, W.; Feng, F. J. Ethnopharmacol. 2017, 209, 175-183. 25. FDA. (2018) https://www.fda.gov/downloads/drugs/ guidancecomplianceregulatoryinformation/guidances/ucm070107.pdf.

26. Zhou, Y. F.; Chen, B. B.; Chen, J. Y.; Dong, Y. W.; Wang, S. H.; Wen, C. C.; Wang, X. Q.; Yu, X. M. Acta Chromatogr. 2018, 30, 131-135.

27. Wu, H. Y.; Yan, Q. Z.; Fan, Z. H.; Huang, M. L.; He, J. M.; Ma, J. S.; Wang, X. Q. Biomed. Chromatogr. 2018, 32, e4255.

28. Wang, S. H.; Lin, Z. X.; Su, K.; Zhang, J.; Zhang, L. J.; Gao, Z. M.; Wang, Z. Y.; Ma, J. S.; Wang, X. Q. Acta Chromatogr. 2018, 30, $26-30$.

29. Shao, L. J.; Jin, Y.; Fu, H. Y.; Ma, J. S.; Wang, X. Q.; Jin, Y. X.; Wen,

C. C. J. Anal. Methods Chem. 2018, $2018,9412708$.

30. Geng, P. W.; Luo, J.; Weng, Z. W.; Fan, Z. H.; Zhang, B.; Ma, J. S.; Wang, X. Q.; Zhang, M. L. Biomed. Chromatogr. 2018, 32, e4273.

31. Chen, L. G.; Zhang, B.; Liu, J. L.; Fan, Z. H.; Weng, Z. W.; Geng,

P. W.; Wang, X. Q.; Lin, G. Y. Biomed. Res. Int. 2018, $2018,1578643$.

32. Chen, L. G.; You, W. W.; Chen, D. W.; Cai, Y.; Wang, X. Q.; Wen,

C. C.; Wu, B. Biomed. Res. Int. 2018, $2018,6562309$.

33. Fang, B. M.; Bao, S. H.; Wang, S. H.; Chen, M. L.; Chen, B. B.; Su, K.; Wen, C. C.; Zhou, Y. F.; Wang, X. Q.; Jin, Y. P. Biomed. Chromatogr. 2017, 31, e3826. 